\title{
e-Phaïstos
}

e-Phaïstos Revue d'histoire des techniques / Journal of the history of technology

II-1 | 2013

Savoirs et sciences sur les machines

\section{François Sigaut, Comment Homo devint faber}

\section{Cyril Lachèze}

\section{OpenEdition}

Journals

Édition électronique

URL : http://journals.openedition.org/ephaistos/1332

DOI : 10.4000/ephaistos. 1332

ISSN : 2552-0741

Éditeur

IHMC - Institut d'histoire moderne et contemporaine (UMR 8066)

\section{Édition imprimée}

Date de publication : 1 juin 2013

Pagination : $97-102$

ISSN : 2262-7340

Référence électronique

Cyril Lachèze, "François Sigaut, Comment Homo devint faber », e-Phaïstos [En ligne], II-1 | 2013, mis en ligne le 15 décembre 2019, consulté le 24 septembre 2020. URL : http://journals.openedition.org/ ephaistos/1332 ; DOI : https://doi.org/10.4000/ephaistos.1332 


\title{
François Sigaut, Comment Homo devint faber (Paris, CNRS, coll. « Biblis », 2012, 236 pages)
}

\author{
Cyril Lacheze \\ Centre d'Histoire des Techniques \\ (CH2ST/EA 127) \\ Université Paris 1 Panthéon-Sorbonne
}

Après une formation et un début de carrière en agronomie, François Sigaut se tourna rapidement vers l'ethnologie et plus particulièrement les aspects techniques de l'agriculture et de l'alimentation, ses domaines de prédilection. Toutefois, son goût pour la remise en cause des idées reçues et trop bien intégrées par la communauté scientifique, qu'il a exercé à propos de la notion de « révolution néolithique » par exemple, s'est associé à son intérêt pour les techniques en général pour produire de nombreuses réflexions plus théoriques. Celles-ci ont abouti à plusieurs articles sur la technologie, et à la dénonciation du désintérêt des sciences humaines pour les techniques. Cette réflexion, prolongée sur la piste de l'action outillée, a abouti à la parution en octobre 2012 de son nouvel ouvrage, Comment Homo devint faber. Celui-ci sera malheureusement son dernier, puisqu'il est soudainement décédé quelques semaines après, le 2 novembre ${ }^{11}$. Bien malgré lui, les théories audacieuses qui y sont avancées peuvent donc être considérées comme une sorte d'aboutissement de sa réflexion dans ce domaine : il n'est donc que justice de se pencher dans les idées, parfois complexes, qui y sont regroupées, pour appréhender au mieux l'œuvre de François Sigaut dans l'étude générale des techniques.

Avant tout chose, il convient de s'arrêter sur la couverture de l'ouvrage. Un titre : Comment Homo devint faber; et une image: la main d'un potier façonnant une céramique en rotation sur un tour. À vrai dire, choix malencontreux de l'éditeur proba- blement, ni l'un ni l'autre ne donnent un aperçu exact du contenu de l'ouvrage et des idées qui y sont développées. François Sigaut ne cherche pas ici à comprendre comment les premiers pré-humains ont réalisé leurs premiers outils, mais plutôt comme ceux-ci ont acquis la «culture » prérequise pour ce faire. «Pourquoi Homo devint faber » aurait ainsi pu paraître plus judicieux, à moins de comprendre faber dans le sens de «capable de fabriquer des outils », sans prendre en compte la concrétisation ou non de cette capacité. De fait, l'image de la céramique n'est pas non plus adaptée: s'il est abondamment question de main dans une partie de l'ouvrage, nous n'y trouvons nulle trace de poterie ou de tours, et d'ailleurs pas plus de mentions d'« industries » préhistoriques ou même de silex (à une exception près), non plus que d'australopithèques ou de toute autre espèce d'Homo que $\mathrm{Sa}$ piens. Les exemples concrets sont systématiquement pris dans le domaine de l'ethnologie, ou des recherches sur les sociétés animales: François Sigaut n'est pas préhistorien, et ne prétend pas l'être. L'Homme est compris dans un sens général et achronique, sans être vraiment défini d'ailleurs, et il n'est pas réellement question des techniques préhistoriques, mais de la Technique en général.

La genèse de l'ouvrage est complexe; le court avant-propos, de François Sigaut lui-même, permet de la comprendre et d'en saisir les implications. Le livre est divisé en trois chapitres : un huitième des pages pour le premier, un quart pour le second, et 
une moitié pour le dernier, sans oublier plusieurs annexes. Les deux premiers sont en réalité issus d'un colloque tenu en 1997, qui n'a jamais été publié (un article, un peu moins détaillé que le livre mais reprenant presque exactement le même développement, est toutefois paru en $2007^{2}$ ). À la suite de Marcel Mauss, l'auteur constatait que les connaissances étaient nettement moins avancées sur les gestes basiques humains que sur ceux des animaux. Surtout, il avançait l'idée que, même lorsque les actions techniques humaines ne faisaient pas intervenir d'outil, elles pouvaient être considérées comme outillées car le corps travaillait sur le modèle d'un outil absent dont il aurait appris à se passer (les mains formant une coupe par exemple) ${ }^{3}$. Pour réaliser cet ouvrage, François Sigaut a repris et développé ses anciennes communications, en y ajoutant une «conclusion » qui a fini par prendre des dimensions gigantesques au fil des réflexions s'accumulant, et qui constitue donc le troisième chapitre du livre. Ce dernier apporte les éléments les plus novateurs de la réflexion de l'auteur sur l'émergence de l'action outillée, les deux premiers ne servant plus, en quelque sorte, que d'introduction.

Le premier chapitre est principalement consacré à l'épistémologie du sujet. L'idée que l'homme soit un animal technique, qui se distingue des autres par sa capacité à s'outiller a cours depuis l'antiquité, mais n'a jamais vraiment été étudiée d'un point de vue scientifique. Revenant sur différentes positions philosophiques quant à l'origine de l'outil, François Sigaut finit par se ranger à l'avis de Bergson et Simone Weil qui font de l'intelligence humaine un produit de la manipulation efficiente des objets, critiquant l'opposition entre intelligence théorique et intelligence pratique. Surtout, François Sigaut insiste sur le fait que ces techniques particulièrement simples sont peu étudiées, peut-être à cause de la nécessité de plonger dans le concret pendant la réflexion. Cette première barrière conduisant à de grosses lacunes dans les données disponibles ne favorise pas l'engagement de nouveaux chercheurs dans cette voie.

La seconde partie de l'ouvrage s'interroge donc sur les manières de sortir de ce "cercle vicieux ». Pour ce qui concerne les données, il existe bien quelques inventaires et classifications, mais peu nombreux, et qui se révèlent mal adaptés : les inventaires simples ne font pas apparaître de signification particulière, et au contraire les classifications raisonnées font souvent appel à une logique arbitraire qui fausse les résultats. Ainsi, le but premier de ce type de recherche doit être l'identification, basée sur un corpus de faits logiques et cohérents, et après définition des niveaux d'analyse. Cette question des niveaux d'analyse se traduit ici par l'emboîtement des concepts de fonctionnement (tenir ou manipuler), de fonction 4 , et de structure. Dans le cas des mouvements de la main qu'étudie François Sigaut, le concept de structure voit s'affronter "anatomistes", ne considérant que la main, et "mécaniciens », ne s'intéressant qu'à l'interaction main-objet. Le premier point de vue, le plus utilisé, donne des possibilités virtuellement illimitées, mais il ne prend pas en compte le fait que seules quelques techniques soient réellement «pertinentes », du fait que les mouvements de la main doivent d'abord être efficaces, et s'individualisent ensuite en fonction des résultats obtenus. La notion d'efficacité fait d'ailleurs l'objet d'un chapitre dédié, qui permet à François Sigaut d'en faire le critère majeur d'une universalité technique indépendante des représentations. Au terme de cette seconde partie, l'auteur arrive donc à la conclusion que l'action technique est toujours outillée, même sans outil ; les « techniques du corps » existent bien mais le corps est alors considéré comme un outil. Au corps et à l'outil, François Sigaut ajoute la matière, qui n'est souvent traitée que comme une abstraction dans la bibliographie.

La troisième partie, la plus novatrice et sur laquelle nous insisterons, s'ouvre donc sur le constat que cet aspect ternaire entre corps, outil et manière fait la spécificité de l'action outillée et est propre aux humains. De fait, l'emploi d'outils est observé 
uniquement chez certains mammifères, la plupart primates, et une centaine d'espèces d'oiseaux, et d'une façon quantitativement minime. François Sigaut introduit donc ici la problématique constituant le titre de son ouvrage : comment est-on passé d'une utilisation très limitée des actions outillées à un usage important ? Il précise cependant immédiatement qu'il n'y a pas de réponse à l'heure actuelle. Selon l'auteur, l'idée de niveaux d'intelligence différents n'a pas de sens, du moins dans l'état actuel des connaissances, puisque cette valeur n'est ni mesurable ni même définie. La distinction principale devrait plutôt être le nombre de situations dans lesquelles chaque espèce fait usage de son " intelligence »: seuls les hommes semblent capables de flexibilité, les animaux n'étant que spécialisés sur certaines pratiques. Ainsi, alors que la distinction homme/animal est bloquée par des positions très clivées, l'action outillée semble une possibilité prometteuse de dépassement, puisqu'elle est connue à la fois chez l'homme et chez les animaux, et de manière comparable.

L'auteur en vient donc nécessairement à se pencher sur l'apparition de l'outil. Les animaux ne perçoivent dans le milieu naturel que les objets pouvant leur être utile; chaque espèce évolue donc dans son propre "monde » composé de son corps et de ce qu'il perçoit spécifiquement. François Sigaut indique que le concept d'outil fait éclater ce modèle, et s'oppose de deux façons à cette définition : c'est un objet artificiel et non naturel, et il se conçoit en dehors d'un monde proche et contingent, de façon réflexive. L'outil est artificiel dans le sens où il s'agit d'une chose neutre à laquelle l'individu a donné une signification. La division entre naturel et artificiel est en réalité contestable, le galet aménagé, par exemple, se trouve à la frontière - mais il faudrait remarquer qu'il s'agit d'une des premières formes d'outil connues : il faudrait plutôt opposer la culture (résultat d'inventions successives) à l'Umwelt (soit, d'après Jacob von Uexküll, le «monde propre » de l'animal). Alors que l'Umwelt est instinctif, la culture passe par un apprentissage. Or, celui-ci, comme pour la marche chez les humains, ne sert chez les animaux qu'à apprendre à mieux réaliser des actions instinctives ; l'intelligence n'est pour eux qu'un moyen, alors qu'elle peut être une fin pour l'homme, au point de l'exercer uniquement pour le plaisir. Dans ce cas, l'intérêt ne serait pas dans le résultat mais dans le fait de réussir. Ceci impliquerait alors un plaisir de la réussite, accessible notamment par les jeux de compétition (et non d'affrontement), qui semblent sinon inexistants du moins rares dans le monde animal. À l'inverse, l'humain qui ne peut exercer son intelligence s'ennuie : le plaisir semble donc un motif pour une activité autonome de l'intelligence. Si François Sigaut note que cette assertion ne peut être prouvée, elle lui paraît être la seule à pouvoir répondre à la problématique.

De plus, les chimpanzés, par exemple, ne vont pas chercher d'eux-mêmes des outils face à un problème, et ne s'en servent que si celui-ci leur est directement présenté : il ne s'agit donc pas d'un "instrument libre». Plus généralement, l'animal porte toute son attention sur le but, en faisant éventuellement intervenir des automatismes, avec des objets le cas échéant. Ceci n'est plus possible dans l'action proprement outillée puisque l'outil devient un but secondaire qui implique de partager l'attention5. Or, puisque l'outil implique la pensée d'une réalité autre que les préoccupations directes, il impliquerait la conscience du soi ; cette conscience proviendrait donc selon François Sigaut du partage de l'attention. Malgré les nombreuses discussions des philosophes, il apparaît finalement que les notions de réel, cause, conscience et réflexion soient totalement interconnectées.

Il reste encore à expliquer l'apparition du plaisir de la réussite. Ceci passe par l'étude du partage de l'expérience, un élément probablement génétique puisque apparaissant extrêmement tôt chez l'enfant. Étant donné que les mutations doivent être sélectionnées comme positives pour être conservées, il convient donc de comprendre en quoi ce partage de l'expérience peut être bénéfique. Celui-ci 
implique une nouvelle forme de lien social, puisque les nouveautés doivent être validées non seulement par l'individu mais par autrui. Ceux-ci partagent donc leur expérience, et le font consciemment: ils savent qu'ils le font et que les autres en ont conscience. Apparaît alors la notion de "sens commun ». François Sigaut y voit la particularité humaine par rapport aux relations interindividuelles présentes dans de très nombreuses sociétés animales. Ce «sens commun » comporte également des conventions et croyances, puisque, au contraire des animaux, l'homme s'en remet à l'expérience d'autrui pour acquérir des informations (éventuellement fausses) sur ce qu'il n'a pu tester lui-même. Une difficulté importante de cette notion résulte du nombre important d'éléments inconscients, donc difficiles à étudier. Différents essais, s'intéressant aux «malentendus culturels », aux " groupes coactifs » (groupes d'individus échangeant entre eux à propos d'une nouveauté), à l'apprentissage, ont montré que le savoir partagé est un identifiant social : l'apprentissage fait entrer l'individu dans le groupe où ce savoir a cours, et un malentendu culturel l'exclut de ce groupe. Ainsi, le partage de l'expérience, en accroissant l'efficacité des actions et l'abondance des ressources, tout en améliorant le lien social, trouve sa synthèse dans le plaisir.

Celui-ci a pourtant été peu étudié, probablement à cause de l'utilitarisme ambiant séparant cognitif et affectif depuis le XIX ${ }^{\mathrm{e}}$ siècle. D'après l'auteur, malgré des critiques régulières, il a fallu attendre les travaux d'Antonio Damasio pour commencer à percevoir une évolution ${ }^{6}$. Pourtant, selon François Sigaut, les éducateurs ont compris depuis longtemps l'importance de l'affectif dans l'apprentissage chez l'enfant. Il faut remarquer que la motivation reste impulsive chez l'animal, quand l'humain passe par une motivation supplémentaire pour le pousser vers un but de manière intelligente. L'auteur suppose d'ailleurs que les cas récents de suicides en entreprise sont liés à la négation de la compétence et de la motivation personnelle, pratique en place depuis le taylorisme ; auparavant, l'exploitation des travailleurs n'impliquait pas forcément de direction technique absolue niant les compétences des travailleurs, notamment dans le cas du travail à domicile.

Il nous reste donc à comprendre comment ce plaisir, si son apparition est biologique, a pu avantager les pré-humains chez lesquels il s'est développé et être donc conservé par la sélection naturelle. Or, les observations effectuées chez les babouins par exemple ont montré que les femelles les plus aptes à trouver des ressources sont les dominées, donc les moins susceptibles de se reproduire. Ces compétences palliant ainsi au fait que les individus dominants accaparent les ressources les plus directement disponibles: ceci montre également que l'intelligence égoïste ne semble pas être une voie vers l'humanisation. Ainsi, chez les primates, les aptitudes sociales, développées par les dominants, semblent incompatibles avec les aptitudes techniques.

François Sigaut se penche également sur les sociétés animales au comportement "eusocial », à savoir principalement les insectes sociaux. Celles-ci ne connaissent pas de domination sociale mais simplement une différenciation de fonctions, qui serait réglée par échange de signaux ${ }^{7}$. Dans ce cas, où la spécialisation de l'individu est physiologique, il est nécessaire de disposer d'échanges entre les individus, non pour les besoins de l'individu mais pour ceux du groupe. À l'inverse de ces "sociétés », celles des mammifères sont en général unies par leur ressemblance et ne peuvent pas échanger. En revanche, il est fréquent qu'ils s'entraident pour effectuer plus efficacement une tâche que l'un pourrait faire seul, mais plus difficilement. Ainsi, l'auteur pose comme hypothèse qu'échange et entraide sont deux types de comportements pouvant potentiellement conduire à des structures sociales «supra-animales », mais en notant que l'échange impliquerait un mode de reproduction ovipare ou des différenciations physiologiques fortes, au contraire de l'entraide qui correspondrait plutôt à des mammifères ou à des espèces sans dimorphisme fort. Les 
deux seules espèces de mammifères dans lesquelles l'échange aurait été observé seraient ainsi le rattaupe, un animal dont l'organisation sociale peut se rapprocher de celle des insectes eusociaux, et l'homme, chez lequel cette pratique a pris une place immense qu'il convient d'expliquer.

François Sigaut ne répond pas directement à cette question, mais commence par remarquer que les termes d'« entraide » et d'« échange », pensés à l'origine pour des humains, ne sont pas forcément très adaptés à la comparaison avec des sociétés animales, et propose à la place les néologismes « homopraxie » (les individus pratiquant la même activité), " hétéropraxie » (activités différentes), et «sympraxie » (la pratique d'une même activité à plusieurs). Les primates pourraient ainsi pratiquer prioritairement l'homopraxie, les insectes l'hétéropraxie, et les interactions comme l'entraide et l'échange seraient des sympraxies, issues selon le cas de l'une des deux configurations précédentes. Or, l'hétéropraxie, menant à l'échange, est peu observée chez les primates en général mais beaucoup chez les hommes, ce qui nous ramène à la problématique de départ. Sigaut reprend ici la réflexion de Pierre Clastres et Claude Lévy-Strauss, proposant l'existence d'une " hérétopraxie primitive » liée à une division sexuelle du travail, suffisamment poussée pour que les célibataires puissent difficilement survivre $^{8}$. Ainsi, chez les humains, les deux sexes sont spécialisés l'un par rapport à l'autre; ce qui n'est pas si surprenant puisque l'hétéropraxie entre mâles et femelles se retrouve dans plusieurs sociétés animales de manière encore plus manifeste que chez l'homme, comme chez les lions pour citer l'une des plus évidentes. Toujours est-il que, selon l'auteur, la répartition sexuelle des tâches, avec le partage de l'attention, serait les deux innovations ayant donné naissance à l'espèce humaine ; on peut donc chercher un éventuel lien entre les deux. L'auteur regrette que la répartition sexuelle des tâches reste peu étudiée dans les études sur l'hominisation. Il rappelle cependant qu'elle était en place au moins depuis l'apparition de l'artisanat, et semble très probable pour des sociétés plus anciennes. François Sigaut suppose donc que partage de l'attention et répartition des tâches étaient en relation dès l'apparition de l'espèce humaine : après l'apprentissage de base à la naissance, commun à de nombreuses espèces et effectué par les parents, un apprentissage plus poussé, propre aux humains, serait effectué par d'autres individus, incluant une distinction sexuelle. Ce second apprentissage permet d'acquérir les savoir-faire propres à chaque sexe afin de devenir des partenaires sexuels attirants, ce qui a pu favoriser l'émergence du plaisir de la réussite. Ceci étant, François Sigaut remarque que, même avec cet avantage, la force semble tout de même trop importante chez les primates pour que ce développement de l'hétéropraxie suffise à lui seul à expliquer ces modifications comportementales.

La conclusion de l'ouvrage, sous le nom de « Remarques finales », permet de mettre au point la réflexion complexe et foisonnante proposée par François Sigaut. S'il n'est pas assuré que l'action outillée soit propre à l'espèce humaine, celle-ci amène à quatre notions fondamentales, à savoir le partage de l'attention, le partage de l'expérience, le plaisir de la réussite et l'échange entre les sexes, les trois premières formant un ensemble indivisible caractéristique de l'espèce humaine. L'origine en est peut-être génétique, notamment pour le plaisir de la réussite. Toutefois, l'action outillée, étant chez les primates caractéristique des dominés exclus de la reproduction, ne produit aucun avantage évolutif. La seule explication à l'émergence de ces processus chez l'homme semble alors être l'échange entre les sexes, l'homme et la femme devenant des partenaires permanents. Notons que ceci n'explique cependant pas comment ni pourquoi cette " innovation » est apparue, ou a été sélectionnée, chez l'homme et uniquement chez lui. Le plaisir pourrait alors provenir de la compétition et de la séduction, ainsi que des avantages produits par la possibilité d'échanger. Ainsi, les premiers humains, plus que d'avoir une intelligence supérieure à celle des autres primates, lui auraient plutôt trouvé de nouveaux 
emplois, en particulier pour la séduction du sexe opposé, et dans lesquels l'action outillée a pu trouver une place de choix.

Comme le note l'auteur lui-même dans les dernières lignes de son ouvrage, cette hypothèse reste très fragile, mais semble être la seule applicable dans l'état actuel des connaissances. Cependant, même avec ces réserves, son intérêt reste évident : l'archéologie, la paléontologie ou les sciences cognitives ne proposent pas encore de réponse réellement solide à cette question du développement sans précédent de l'action outillée chez l'homme, faute d'éléments fiables. Si, malgré cette difficulté, l'on souhaite se pencher sur cette problématique, le recours à l'ethnologie voire à la philosophie, semble donc une idée intéressante et prometteuse, malgré le fait que le manque d'intérêt ou encore d'inventaires, souligné par l'auteur, puisse parfois nuire à la réflexion. Afin de préciser cette piste, qui reste pour l'instant très axée sur une recherche purement intellectuelle, il nous semblerait ainsi bienvenu de l'ancrer de nouveau dans un aspect plus matériel : nous avons signalé d'emblée que l'ouvrage ne comprend ni résultat d'études liées aux sciences cognitives, ni description d'outil préhistorique, ni même de nom d'espèce d'Homo autre que Sapiens. Il nous semblerait donc intéressant de croiser la réflexion développée par François Sigaut tout au long de sa carrière et dans cette ultime œuvre, avec les dernières découvertes des sciences directement intéressées par les problématiques qui y sont développées : ceci permettrait sans doute de faire progresser à la fois la compréhension théorique de ces mécanismes et de leurs manifestations concrètes, pour aboutir, à terme, à une lecture globale du phénomène.

${ }^{1}$ ARCHAMBAULT DE BEAUNE, Sophie, «Hommage à François Sigaut », Carnets de la MAE, décembre 2012. En ligne sur le site des Carnets de la MAE, consulté le 19/03/2013: http://mae.hypotheses.org/358
2 SIGAUT, François, «Les outils et le corps », Communications, 81, 2007, pp.9-30.

${ }^{3}$ D'après l'auteur, cette formulation se place donc à l'inverse du sens commun et de théories comme celles de la projection organique d'Ernst Kapp (1877), selon laquelle l'outil serait en quelque sorte dérivé du corps.

${ }^{4}$ Divisé en sept classes : locomotion, manutention, lancer, façonnage direct, façonnage outillé, toucher, faire signe.

${ }^{5}$ François Sigaut précise qu'aucune recherche poussée n'a cependant été portée sur cette question, à part quelques esquisses très rapides. Il note cependant, notamment avec Maurice Pradine, que la notion de cause, absente chez l'animal, est technique et définit la pensée humaine. Cet auteur, très rapidement oublié par la suite, ne pose cependant pas la question entre cette notion de cause et l'intelligence.

6 DAMASIO, Antonio, "L'Erreur de Descartes. La raison des émotions », Paris, Odile Jacob, 1995, 368 p.

7 Il faut toutefois noter que la différenciation des castes est au moins en partie définie d'une part génétiquement, et d'autre part par un nourrissage différencié des larves.

8 CLASTRES, Pierre, "Archéologie de la violence. La guerre dans les sociétés primitives ", Libre, 1, 1977, pp.137-173. 